\title{
Molecular Particularity in Rare Tumour of Buttock: Case Report and Literature Review*
}

\author{
El Fatemi Hinde $^{1 \#}$, Florence Mishellany ${ }^{2}$, Pierre Gimbergues ${ }^{3}$, Frédérique Penault-Llorca ${ }^{2}$ \\ ${ }^{1}$ Department of Pathology, Hassan II Teaching Hospital, Fez, Morocco; ${ }^{2}$ Department of Pathology, Centre Jean-Perrin, Cler- \\ mont-Ferrand, France; ${ }^{3}$ Department of Surgery, Centre Jean-Perrin, Clermont-Ferrand, France. \\ Email: "hinde0012@hotmail.com
}

Received June 26 ${ }^{\text {th }}$, 2012; revised July 27 ${ }^{\text {th }}$, 2012; accepted August $17^{\text {th }}, 2012$

\begin{abstract}
Introduction: Hyalinizing spindle cell tumor with giant rosettes (HSCT) is a very uncommon mesenchymal tumor that has similar morphological and biological features to the low-grade fibromyxoid sarcoma (LGFMS). Case Report: Reported herein is a case of primary tumour of buttock HSCT that had rare FUS-CREB3L1 fusion transcripts, a product of characteristic chromosomal abnormality t $(7,16)(\mathrm{q} 33, \mathrm{p} 11)$ of HSCT and LGFMS. The patient was a 48-year-old man who had a large solitary mass in the buttock. Histologically, it was composed of bland spindle cells with variable cellularity deposited in a densely hyalinized stroma alternating with myxoid areas. Characteristic collagen rosettes were scattered in the cellular areas. Reverse transcription-polymerase chain reaction (RT-PCR) assay using formalin-fixed, paraffin-embedded tissue detected FUS-CREB3L1 fusion transcripts. In our knowledge is the second case may display a variant FUS/CREB3L1 fusion transcript in international literature. Conclusion: LGFMS and HSCT probably have a wider spectrum of morphologic features than previously thought, the awareness of which will help pathologists to avoid diagnostic pitfalls. Demonstration of the $t(7,16)$ translocation will help to diagnose difficult cases with unusual histologic features.
\end{abstract}

Keywords: Deep Soft Tissue; Fibromyxoid Sarcoma; Hyalinizing Spindle Cell Tumor; RT-PCR; Fusion Transcripts; CREB3L1

\section{Introduction}

Hyalinizing spindle cell tumor with giant rosettes (HSCTGR) is a recently described low-grade sarcoma with a deceptively benign histological appearance [1]. Because it has similar clinicopathological features and biological behavior to that of low-grade fibromyxoid sarcoma (LGFMS), HSCT might be regarded as a variant of LGFMS [2,3]. Low-grade fibromyxoid sarcoma (LGFMS) is a rare soft-tissue tumor with a deceptively benign histologic appearance affecting predominantly young adults during the fourth decade of life [1]. Lowgrade fibromyxoid sarcoma has a predilection for involving deep soft tissues of the thigh, inguinal region, or chest wall, affecting less frequently the shoulder or axilla [1]. Local postsurgical recurrence and metastases to lungs and bone are frequently seen [1]. Additionally, both tumors have a characteristic FUS-CREB3L2 fusion gene transcript. A product of reciprocal translocation, $\mathrm{t}(7$, 16) (q33, p11), further supporting this concept $[4,5]$. HSCT arises in a variety of deep soft tissues, most com-

*The authors declare no conflict of interest.

${ }^{\#}$ Corresponding author. monly in the extremities. We describe here a new case of primary tumour of buttock HSCT harboring a molecular particularity with a rare FUS- CREB3L1 fusion transcripts.

\section{Case Report}

A 48-year-old man presented with a 13-cm painful left buttock mass that had been present for 1 year. The mass was palpable by digital rectal examination but did not involve the mucosa. A superficial FNAB of the subcutaneous mass was performed and diagnosed as "spindle cell neoplasm, favor low grade sarcoma”. The lesion was resected with negative surgical margins. Macroscopically, the tumors were well circumscribed, firm, and yellow-tan. For microscopic examination, wet formalin-fixed tissue was available in our case. Microscopically, a pseudocapsule surrounded the tumors. The tumors were comprised of bland, fibroblastic spindle cells arranged in a whirling growth pattern within a hyalinized collagen bundles and a myxoid matrix (Figure 1). The myxoid areas were characterized by low cellularity with a prominent capillary network. However, in some areas the 


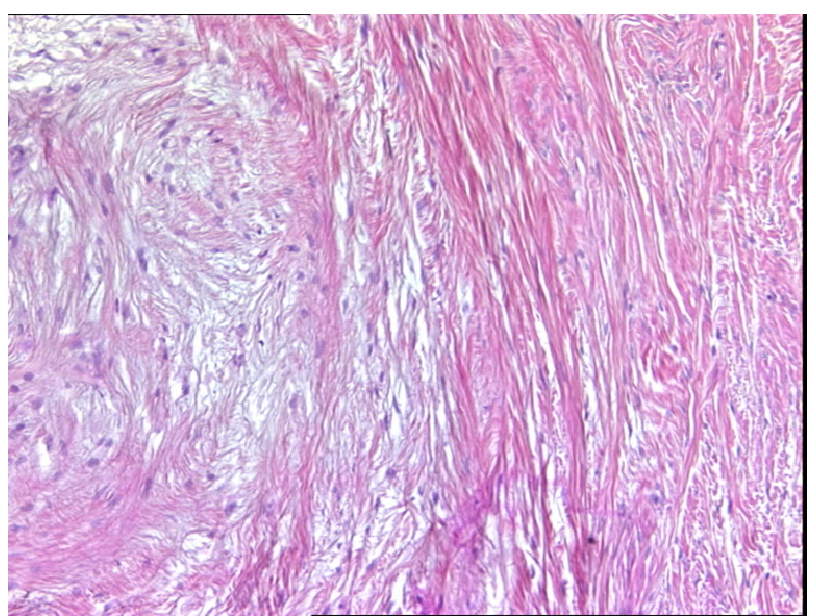

Figure 1. formalin-fixed tissue with (hematoxylin eosin and safran coloration). HES $\times 10$. Histologic appearance showing fusocellulaire proliferation of heterogeneous density.

spindle cells were concentrated around blood vessels. Alternating with the myxoid regions were fibrous areas with varying degrees of cellularity, including some hypocellular areas comprised nearly entirely of dense collagenous tissue. A large rosette-like structure with hyalinized stroma was found (Figure 2), which is characteristic of LGFMS. Immunohistochemistry in our case revealed strong positivity with vimentin only; no staining was observed with Smooth muscle actin, desmin, protein S-100, or CD34 in the sections examined. PCR amplifycations were performed in a 50-lL reaction volume containing 13 AccuPrime Pfx reaction mix, 1 unit AccuPrime Pfx DNA polymerase, $0.3 \mathrm{lM}$ of each of the forward and reverse primers and $200 \mathrm{ng}$ template DNA. The PCR was run on a PCT-200 DNA Engine. The cycling included an initial denaturation at 958C. For 2 min, followed by 30 cycles of $15 \mathrm{~s}$ at $958 \mathrm{C}, 30 \mathrm{~s}$ at 588C, and 2 $\min$ at $688 \mathrm{C}$, and a final extension for $5 \mathrm{~min}$ at $728 \mathrm{C}$. All ligations were performed overnight at $168 \mathrm{C}$ in 10-lL reaction volume containing 13 Ligase Reaction Buffer, 5 units T4 DNA ligase, and 1:3 vector to insert ratio. When purification was required, the DNA fragments were purified using either the QIAquick gel extraction kit or the QIAquick PCR purification kit. For sequence analysis, the ABI Prism BigDye terminator v1.1 cycle sequencing kit was used and the products were analyzed on an Applied Biosystems Model 3100-Avant DNA sequencing system. RT-PCR demonstrated the FUS-CREB3L1 fusion transcripts resulting from the reciprocal translocation, $\mathrm{t}(7,16)$ (q33, p11). The patient received no additional therapy. Clinical follow-up, including radiographic studies, had not detected recurrent tumor at 3 years of follow-up.

\section{Discussion}

The HSCTGR was first reported in a series of 19 cases

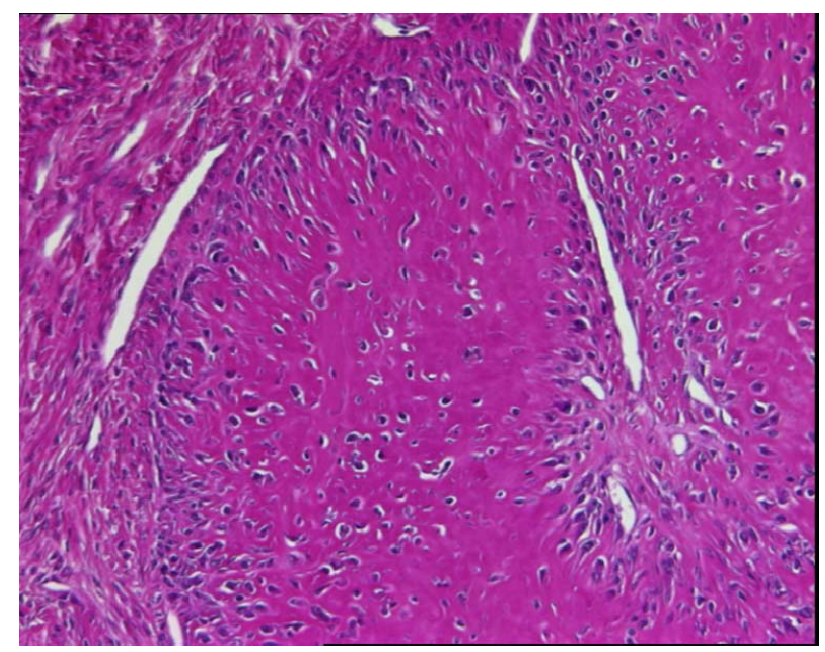

Figure 2. Formalin-fixed tissue with (hematoxylin eosin and safran coloration). HES $\times$ 40. A large rosette-like structure with hyalinized stroma.

culled from a large soft tissue consultation service by Lane et al. in 1997 [2]. Clinically, HSCTGR can develop at almost any age (average age, 38 year) in the deep soft tissues of the extremities, particularly the thigh $[2,6]$. Other sites of involvement are the chest wall, axilla, rarely buttock, and the [2]. The most common symptom is a painless, deep-seated, slowly enlarging mass $[2,6]$. Grossly, the tumor is an oval multilobulated mass, ranging in size from 2 to $20 \mathrm{~cm}$ in diameter. Although most of the lesions appear well circumscribed, they can extensively infiltrate the surrounding soft tissue [6,7]. A prior report on the imaging characteristics of LGFMS described a heterogeneously hyperechogenic multi-nodular sonographic appearance, a heterogeneous MR imaging appearance with low to slightly high SI on T1-weighted images, heterogeneously low to high SI on T2-weighted images, and heterogeneous postcontrast enhancement [3]. The cut surface has a whorled white-tan appearance. Cystic degeneration is an uncommon finding [7]. Histologically, the tumor cells have a deceptively bland appearance, consisting of short, ill-defined fascicles of fusiform to spindled cells with minimal atypia and barely perceptible levels of mitotic activity [6]. In a small number of cases there may be foci that are more cellular and atypical, which would mimic an intermediate grade fibrosarcoma [3,6]. The mitotic figures were difficult to identify, usually with less than one mitosis per 50 high-power fields [6]. The most characteristic feature was the presence of a variable number of large rosettes like structures. These structures either occupy only a small portion of the lesion or are so prominent as to obscure the other features of the neoplasm [2,6]. The rosettes, which tended to cluster, were made up of a central collagenous core flanked by an irregular rim of rounded neoplastic cells. The immunohistochemical profile, espe- 
cially in HSCTGR, has suggested a neural phenotype, with the cells forming the rosettes being Leu-7, S-100, and pgp 9.5 positive and the spindle cells usually being negative $[2,6,7]$. The significance of the rosettes is unclear. However, cells forming rosettes have the ultrastructural features of fibroblasts [6]. Moreover, they appear to be part of the neoplasm rather than a necrobiotic granuloma-like response within the tumor [6]. Similar appearing giant collagen-containing rosettes may be seen in other mesenchymal tumors, including a neuroblastoma-like neurilemmoma, in which the rosettes are comprised of a core of collagen, flanked by small, round, differentiated Schwann cells [6]. The microscopic differential diagnoses of LGFMS include low-grade myxofibrosarcoma, myxoid neurofibroma, and myxoid solitary fibrous tumor. Low-grade myxofibrosarcoma has more cellular atypia and less swirling of tumor cells in a uniformly myxoid stroma. Myxoid neurofibroma shows more wavy nuclei of the spindle cells and strong S-100 positivity. The osteoid, in rare cases of osteosarcoma, may be confused for the collagen rosettes. Calcification in the material and the surrounding marked nuclear pleomorphism will help in settling the issue $[2,8]$. In the original report by Lane et al., follow-up information was available for 12 of the 19 patients, with a mean follow-up period of 39 months [2,6]. Of the 12 patients, 7 were treated with a simple local excision and 5 by a wide excision. Although one of the patients treated by simple excision developed a local recurrence 20 months after the initial surgery, none of the remaining patients had a recurrent or metastatic disease within the follow-up period. Therefore, this lesion was considered to be a low-grade sarcoma, and a wide surgical excision is the treatment of choice [6]. Though the lesion appears bland in morphology, long-term follow-up has revealed that it has a potential for metastasis, especially to the lungs $[3,7,9]$. Improved recognition and treatment have improved the prognosis of the lesion but, nevertheless, prolonged follow-up is necessary. The discovery that LGFMS has a specific translocation $\mathrm{t}(7,16)$ (q33, $\mathrm{p} 11)$, or in rare cases $\mathrm{t}(11,16)$ (p11, p11), has greatly facilitated the diagnosis [10]. Through these translocations, the chimeric genes FUS-CREB3L2 or FUS-CREB3L1, respectively, are created. At the molecular level, the 5'-part of FUS, encoding a transactivation domain, is fused to the 3'-part of CREB3L2 or CREB3L1, encoding a basic leucine zipper (bZIP) DNA-binding domain [10]. A subset of LGFMS cases expresses the FUS-CREB3L2 fusion transcript but lacks the typical $t(7,16)$ and instead harbors a supernumerary ring chromosome, which may contain the fusion gene, as the sole aberration [1,6]. Karyotypic information on LGFMS reveals few other recurrent aberrations, suggesting that the chromosomal translocations are tumorigenic events.
In conclusion, we report a case of primary HSCT arising in the buttock harboring a molecular particularity with a rare FUS-CREB3L1 fusion transcripts. The recognition of typical histological features and behavior of HSCT of the soft tissue would be helpful for the management of patients, and detection of the characteristic FUS-CREB3L2 fusion transcript or rarely FUS-CREB3L1 is necessary for the differential diagnosis of HSCT involving unusual site. Therefore, an accurate tissue diagnosis with wide surgical excision and a close follow-up are essential.

\section{Acknowledgements}

The authors thank Prof. J. M. Coindre for its opinion and diagnostic follow-up study in molecular biology.

\section{REFERENCES}

[1] G. P. Nielsen, M. K. Selig, J. X. O’Connell, S. B. Keel, G. R. Dickersin and A. E. Rosenberg, "Hyalinizing Spindle Cell Tumor with Giant Rosettes: A Report of Three Cases with Ultrastructural Analysis,” American Journal of Surgical Pathology, Vol. 23, No. 10, 1999, pp. 1227-1232.

[2] K. L. Lane, R. J. Shannon and S. W. Weiss, "Hyalinizing Spindle Cell Tumor with Giant Rosettes. A Distinctive Tumor Closely Resembling Low-Grade Fibromyxoid Sarcoma," American Journal of Surgical Pathology, Vol. 21, No. 12, 1997, pp. 1481-1488.

[3] A. L. Folpe, K. L. Lane, G. Paull and S. W. Weiss, "Low-Grade Fibromyxoid Sarcoma and Hyalinizing Spindle Cell Tumor with Giant Rosettes. A Clinicopathologic Study of 73 Cases Supporting Their Identity and Assessing the Impact of High-Grade Areas,” American Journal of Surgical Pathology, Vol. 24, No. 10, 2000, pp. 13531360.

[4] R. Reid, C. Silva, L. Paterson, E. Ryan and C. Fisher, "Low-Grade Fibromyxoid Sarcoma and Hyalinizing Spindle Cell Tumor with Giant Rosettes Share a Common $\mathrm{t}(7,16)(\mathrm{q} 34, \mathrm{p} 11)$ Translocation," American Journal of Surgical Pathology, Vol. 27, No. 9, 2003, pp. 1229-1236.

[5] A. Matsuyama, M. Hisaoka, S. Shimajiri, et al., "Molecular Detection of FUS-CREB3L2 Fusion Transcripts in Low-Grade Fibromyxoid Sarcoma Using FormalinFixed, Paraffin-Embedded Tissue Specimens,” American Journal of Surgical Pathology, Vol. 30, No. 9, 2006, pp. 1077-1084.

[6] S. W. Weiss and J. R. Goldblum, "Enzinger and Weiss's Soft Tissue Tumors," 4th Edition, Mosby, St. Louis, 2001, pp. 431-435.

[7] S. E. Vernon and P. A. Bejarano, "Low Grade Fibromyxoid Sarcoma: A Brief Review," Archives of Pathology \& Laboratory Medicine, Vol. 130, No. 9, 2006, pp. 1358-1360.

[8] T. Hansen, K. Katenkamp, M. Brodhun and D. Katenkamp, "Low-Grade Fibrosarcoma-Report on 39 Not Otherwise Specified Cases and Comparison with Defined Low-Grade Fibrosarcoma Types,” Histopathology, Vol. 
39, No. 4, 2006, pp. 152-160.

[9] J. M. Woodruff, C. R. Antonescu, R. A. Erlandson and P. J. Boland, "Low Grade Fibrosarcoma with Palisaded Granulomalike Bodies (Giant Rosettes): Report of a Case That Metastasized," American Journal of Surgical Pathology, Vol. 23, 1999, pp. 1423-1428. doi:10.1097/00000478-199911000-00015
[10] F. Mertens, C. D. Fletcher, C. R. Antonescu, J. M. Coindre, M. Colecchia, et al., "Clinicopathologic and Molecular Genetic Characterization of Low-Grade Fibromyxoid Sarcoma, and Cloning of a Novel FUS/CREB3L1 Fusion Gene,” Laboratory Investigation, Vol. 85, No. 3, 2005, pp. 408-415. doi:10.1038/labinvest.3700230 